\title{
The Effective Two-Particle Interaction of Cold Atoms as Derived from Bragg Scattering
}

\author{
R. Sarjonen · M. Saarela $\cdot$ F. Mazzanti
}

Received: 4 May 2012 / Accepted: 12 September 2012 / Published online: 28 September 2012

(C) Springer Science+Business Media New York 2012

\begin{abstract}
We study the dynamic structure function of ultracold alkali-metal gases for large scattering lengths and momenta where corrections to the mean field approximation become important. We compare our result with the Bragg-scattering measurements in ${ }^{85} \mathrm{Rb}$ by Papp et al. (Phys. Rev. Lett. 101:135301, 2008) and show that these experiments set very strict limits to the shape of the effective two-particle interaction ruling out the contact and hard spheres potentials. Using the Feshbach resonance approximation we derive the effective interaction, which turns out to be very similar to the soft spheres potential in momentum space. At large scattering lengths the interaction becomes universal and could be directly measured by Bragg scattering. We also discuss the experimental conditions needed for the appearance of the maxonroton structure in the excitation spectrum and finally show that when the scattering length becomes larger than 2000 Bohr radii the uniform gas phase undergoes a phase transition into the density wave state.
\end{abstract}

Keywords Ultracold Bose gases · Feshbach resonance · Bragg scattering · Dynamic structure

\section{Introduction}

Cold atoms have been very successful in providing testing ground for theories of weakly interacting quantum gases. In a typical BEC experiment the separation distance between atoms is long, collisions between them are low in energy and thus

R. Sarjonen $\cdot$ M. Saarela $(\varangle)$

Department of Physics, University of Oulu, P.O. Box 3000, 90014 Oulu, Finland

e-mail: Mikko.Saarela@oulu.fi

F. Mazzanti

Dep. de Fisica i Enginyeria Nuclear, Universitat Politecnica de Catalunya, Campus Nord B4-B5,

08034 Barcelona, Spain 
only s-wave scattering theory is needed to model them. Under these circumstances, the key parameter in the theory is the scattering length $a$, which determines the scattering amplitude in the zero energy limit and sets the main length scale of the system. It is customary to assume that the range of the interaction between atoms is short in this scale and model the system with the contact interaction,

$$
V_{\text {cont }}(\tilde{\mathbf{r}})=4 \pi \frac{\hbar^{2}}{m a^{2}} \delta(\tilde{\mathbf{r}}) .
$$

The scattering length defines then also the energy scale $\hbar^{2} /\left(m a^{2}\right)$ when the separation distance is expressed in units of the scattering length $\mathbf{r}=\tilde{\mathbf{r}} a$.

In the many-body system the second parameter is the density $\rho$ and it is also expressed in units of the scattering length through the dimensionless gas parameter $x=\rho a^{3}$. In dilute, weakly interacting systems $x$ is very small, typically $x<0.0001$, which means that the average separation distance between particles is more than ten times the scattering length. Under such conditions the ground state energetics can be expanded using the Lee-Huang-Yang (LHY) expansion $[1,2]$ in powers of $x$.

Excitations of dilute Bose gases are well described by the Bogoliubov theory when the energy and momentum transfers are low, typically when $k \ll 1 / a$. The speed of sound of the sound mode is $c=\sqrt{4 \pi x} \hbar /(m a)$, which is verified experimentally [3-6]. At large momentum transfers the excitation spectrum is dominated by the single-particle excitations $\hbar^{2} k^{2} /(2 m)$. The interesting region lies between those two limits.

In strongly correlated quantum fluids like liquid ${ }^{4} \mathrm{He}$, the hard sphere Bose gas or even in the charged Bose gas, the elementary excitation spectrum has the typical maxon-roton shape, but of course its appearance for a given interaction depends on the density. In liquid ${ }^{4} \mathrm{He}$ the scattering length $a=104 \AA$ is much larger that the separation distance between particles $d \sim 3.5 \AA$ as determined from the binding energy of a very weakly bound dimer state of $1.1 \mathrm{mK}$ [7] and the high liquid density $0.0218 \AA^{-3}$ giving a huge value to the gas parameter $x \sim 25000$. Another point of view is to ignore the fact that ${ }^{4} \mathrm{He}$ is a liquid and treat it as a system of the hard spheres Bose gas with the radius of the sphere $\sim 2.2 \AA$. If that is set equal to the scattering length [8-10] and then the liquid ${ }^{4} \mathrm{He}$ density corresponds to $x \sim 0.23$. For the system of hard sphere Bosons the roton minimum appears when $x>0.15$ and the gas solidifies at $x \sim 0.25$.

The existence of the roton minimum in the excitation spectra of cold gases has recently been under intensive speculations especially in dipolar gases [11-13]. For quite general soft core interactions interesting suggestions are made that softening of the roton mode could lead to a clustered supersolid phase [14-16] or a density wave state [17]. The models used for the two-particle interactions range from hard spheres to soft spheres with or without the $1 / r^{3}$ or $1 / r^{6}$-tails. In the experimental side the bending of the Bragg scattering line shifts [18] and the bending of the critical temperature of the superfluid transition $[9,19]$ as a function of $x$ has been connected to the maxon-roton behavior.

In this paper we analyse the Bragg scattering measurements of the dynamic structure function in ${ }^{85} \mathrm{Rb}$ [18]. The measurements have been carried out above the $B_{0}=154.9 \mathrm{G}$ resonance magnetic field for the momentum transfer $k=4 \pi / 780 \mathrm{~nm}$ 
and the density $\rho=7.6 \times 10^{13} \mathrm{~cm}^{-3}$, with the scattering length spanning the range $153 a_{0}<a<891 a_{0}$ with $a_{0}$ the Bohr radius. This tests the regions where $k a<0.8$ and $x<0.01$, which are beyond the validity of the mean field approximation. The results show a maximum in the excitation spectrum as a function of the scattering length and attempts have been made to explain that in the framework of Beliaev's formulation of the many-body theory $[18,20,21]$.

We show, following the methods presented in Refs. [17, 22], that Bragg scattering experiments strongly limit the possible shapes of the effective two-body potentials, ruling out the hard spheres and the contact interaction models and favoring the soft spheres models. In order to make firm statements about the two-body interaction we use the non-perturbative many-body method based on Jastrov correlations [23], which is valid from a dilute gas limit up to solidification densities. We assume that the effective two-body potential scales in the scattering length units - as do the hard sphere and contact potentials-, because the Feshbach resonance dominates the interaction at large scattering lengths. In this way, the interaction becomes universal at the unitarity limit where $a \rightarrow \infty$. We show new results for the ${ }^{133} \mathrm{Cs}$ resonance at $B_{0}=-11.7 \mathrm{G}[24,25]$, because there the dimer binding energies are experimentally determined.

The critical parameter for the roton minimum to appear and a possible phase transition to take place is the gas parameter $x$. The increase of the density of condensed atoms in the center of the trap is limited, because the gas of cold atoms is inherently metastable and the instability increases because of three-particle recombination, with a rate proportional to $x^{2} / a^{2}$ [26]. The bound states are then lost from the condensate and the density decreases. Another option to increase the gas parameter is to increase the scattering length by changing the binding energy of a weakly bound state via the external magnetic field. In order to describe these tunable Feshbach resonance systems realistically one needs a model with at least two coupled states with different magnetic moments. One of the states is the bound, closed channel, and the other one is the open channel $[27,28]$. They form, in momentum space, a set of coupled Lippmann-Schwinger equations that define the scattering T-matrix. The Feshbach approximation [29], in which only the dominant resonance state is taken into account, provides the simplest way to solve the T-matrix equation. Typically the bare interaction between atoms chosen in those equations is the contact interaction, which is a constant in momentum space. The well known problems with the large momentum divergence are cured with a cut-off function. The parameters of that function are fitted to measured scattering data, binding energies and magnetic properties using renormalization. In a sense this is equivalent to using a parametrized two-body interaction in momentum space.

The true atom-atom potential has a large number of bound states. The problem is to derive from the T-matrix the long wavelength part of the effective two-body potential in the single, scattering channel that makes the gaseous phase stable. It is clear that if the two-, three- and higher-body clusters are bound with that potential then the gas phase is not the ground state. However, one can still have a metastable gas, which lives long enough for experiments, if the interaction has a repulsive enough tail at large distances besides the attractive part closer to the origin causing the binding. For a metastable system the short-ranged attraction can then be cut off from the effective 
two-body potential in the many-body calculations of the homogeneous atomic gas. That is in essence how we derive the interaction in this work.

In Sect. 2 we analyse the measurement of the dynamic structure function of Bose gases using correlated basis function theory (CBF) [23, 30, 31]. In the Feynman theory of excitations the dynamic structure function has just one pole at the energy

$$
\varepsilon(k)=\frac{\hbar^{2} k^{2}}{2 m S(k)}
$$

with $S(k)$ the static structure function. The CBF prediction includes multiphonon contributions and we show how they separate from the elementary excitations peak, increasing the theoretical width in energy. However in actual experiments the limited instrumental resolution does not show such details in the structure, which explains the missing fraction of the width in Ref. [18].

The elementary excitations spectra and Bragg scattering line shifts are calculated in Sect. 3 for the hard and soft spheres potentials. We show that they lead to completely different results for the line shifts and that, as a consequence the hard spheres potential can be ruled out at large scattering lengths. We extend the experimentally available region up to $x \sim 0.2$ and show how the maxon-roton structure appears in both systems with increasing $x$, although the physical mechanisms producing it are different: in the case of hard spheres the system is approaching the solid phase, whereas the soft spheres system remains in the gas phase, but becomes unstable against the formation of a density wave state.

In Sect. 4 we derive the s-wave phase shifts from the renormalized T-matrix equation following Refs. [17, 22, 32]. The effective two-body interaction is then solved from the inverse scattering problem using the Marchenko equations [33, 34] in Sect. 5. At the unitarity this interaction becomes universal. With this potential we get good agreement to the Bragg scattering line shifts and, surprisingly, to the soft spheres potential in momentum space. The critical parameters for the phase transition to the density wave state are also evaluated.

\section{Dynamic Structure Function}

The dynamic structure function $S(k, \omega)$ contains all the information of excitations modes of a homogeneous Bose system. At low momenta and frequencies, typical Bose fluids show a sharp peak which defines the elementary excitation mode. At higher frequencies multiphonon excitations form a broad continuous distribution. A suitable framework for calculating $S(k, \omega)$ is provided by the CBF theory [30], which describes very well weakly interacting Bose gases [35-37] and yields reasonable results also for such strongly interacting systems as liquid ${ }^{4} \mathrm{He}$ [31]. That theory leads to a dynamic linear response function of the general form

$$
\chi(k, \omega)=\frac{S(k)}{\hbar \omega-\varepsilon(k)-\Sigma(k, \omega)}-\frac{S(k)}{\hbar \omega+\varepsilon(k)+\Sigma^{*}(k,-\omega)},
$$

where $\varepsilon(k)$ is the Feynman excitation spectrum, and $\Sigma(k, \omega)$ is the phonon selfenergy arising from phonon-splitting and recombination processes. The details of the 
Fig. $1 S(q, \omega)$ for hard sphere Bosons at $x=0.1$. Frequencies and momenta are in units of $\hbar / m a^{2}$ and $1 / a$, respectively (Color figure online)

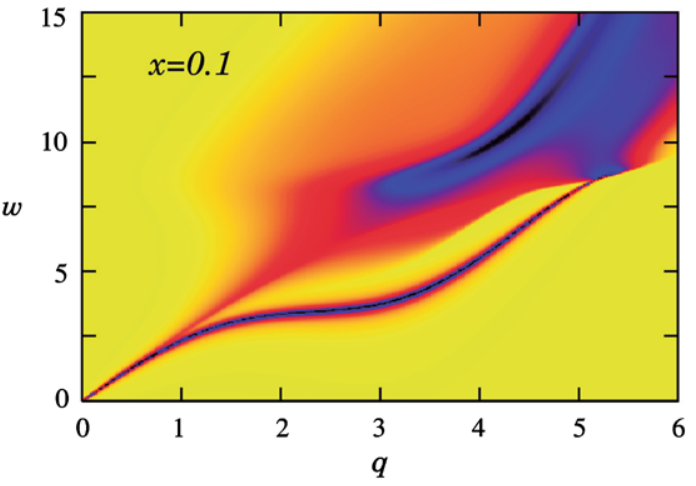

derivation of the self energy are given in Refs. [17, 35]. The imaginary part of the linear response defines the dynamic structure function for positive frequencies,

$$
S(k, \omega)=-\frac{1}{\pi} \Im m[\chi(k, \omega)] .
$$

A typical picture of $S(k, \omega)$ for a strongly interacting Bose system is shown in Fig. 1, which is calculated for a gas of hard spheres at $x=0.1$. At such large values of $x$ the elementary excitation mode separates well from the multiphonon contributions. Yet, $x$ is not high enough for a clear roton minimum to form as in ${ }^{4} \mathrm{He}$. Although it is an order of magnitude larger than what is experimentally available at the moment in cold Bose gases. The elementary excitation pole becomes visible in the theoretical study when $x \sim 0.01$. This structure should be compared with the Feynman approximation, which includes only the elementary excitation pole, yielding the approximation

$$
S(k, \omega)=S(k) \delta(\varepsilon(k)-\hbar \omega) .
$$

In the Bragg scattering experiments of Ref. [18] $x<0.01$ and the elementary excitations and multiphonon contributions are merged together. This is seen in Fig. 2, which shows results of the $\mathrm{CBF}$ calculation at two different scattering lengths $354 a_{0}$ and $811 a_{0}$ corresponding to $x=0.0005$ and $x=0.006$, respectively, for a system of Bosons interacting through the soft spheres potential $[17,36]$

$$
V(r)= \begin{cases}V_{0}, & r \leq R \\ 0 & r>R\end{cases}
$$

with $R=3.5 a$ and $V_{0}=0.106158 \hbar^{2} /\left(m a^{2}\right)$. The parameters are chosen such that they always give the desired value of the two-body, s-wave scattering length $a$. The only free parameter then is the radius, which we fit to the Bragg scattering experiments. Figure 2 also shows the $\operatorname{CBF} S(k, \omega)$ and the Feynman approximation, both folded with the instrumental resolution (IR) function of the experiments [18]. The maxima of these broadened distributions agree very well at low scattering lengths, but at the largest experimental scattering lengths a tiny shift of $\sim 0.3 \mathrm{kHz}$ to lower 

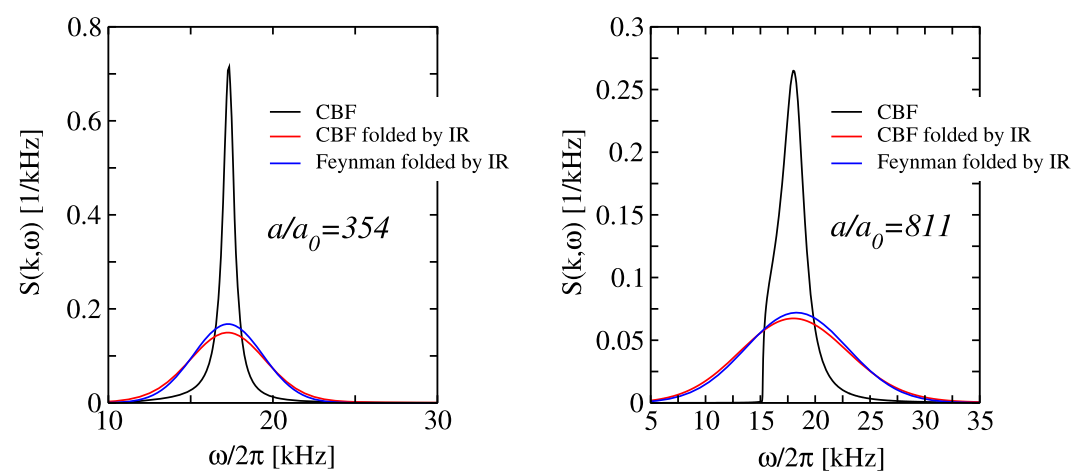

Fig. $2 S(k, \omega)$ calculated at the experimental momentum $k=4 \pi / 780 \mathrm{~nm}$ and density $\rho=7.6 \times$ $10^{13} \mathrm{~cm}^{-3}$ using the soft spheres potential and the marked scattering lengths. Comparison in made between the CBF $S(k, \omega)$ (highest peak with black line), the $\operatorname{CBF} S(k, \omega)$ folded with the instrumental resolution functions of Ref. [18] (lowest peak with red line), and the Feynman approximation also folded with these functions (blue line) (Color figure online)

Fig. 3 The CBF $S(k, \omega)$ calculated at the experimental momentum and density for soft spheres with different scattering lengths shown in the figure (Color figure online)

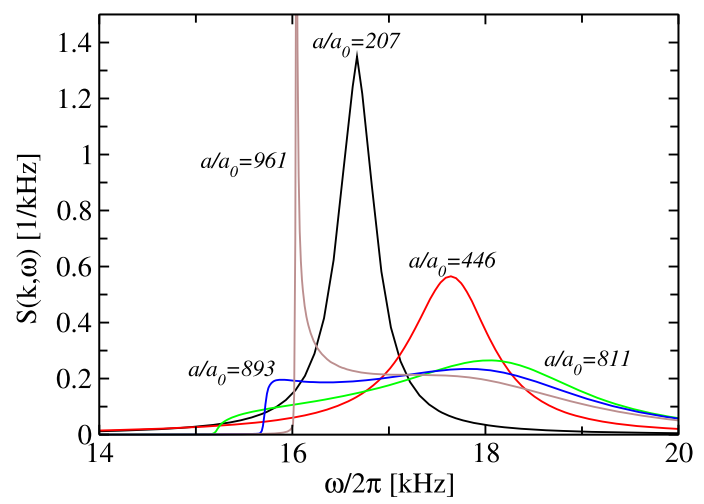

frequencies obtained by using the full $\mathrm{CBF}$ theory becomes barely visible. This feature appears because the CBF distributions become more and more asymmetric with increasing $a$ and the multiphonon contributions begin to separate from the elementary excitation pole. These features are shown in Fig. 3 for a set of distributions calculated at measured scattering length values. In experiments the width of multiphonon contributions is seen, as in Fig. 2, convoluted with the IR function. A slight increase of $0.4 \mathrm{kHz}$ in the width caused by the broader distribution of CBF results is observed at the highest $a$ in rough agreement with experiments. From this analysis we conclude that the relevant quantity describing the dynamics within the experimental resolution is $S(k)$ through the Feynman approximation of Eq. (5).

The fact that the peaks of the CBF and Feynman approximations coincide once broadened by the (fairly wide) IR function can be traced back to the lowest order energy-weighted sum rules fulfilled by the dynamic structure function [23], the lead- 
ing ones being

$$
\begin{aligned}
& m_{0}(k)=\hbar \int_{0}^{\infty} d \omega S(k, \omega)=S(k), \\
& m_{1}(k)=\hbar^{2} \int_{0}^{\infty} d \omega \omega S(k, \omega)=\frac{\hbar^{2} k^{2}}{2 m},
\end{aligned}
$$

which imply that the average value of the frequency

$$
\langle\omega(k)\rangle=m_{1}(k) /\left(\hbar m_{0}(k)\right)=\hbar k^{2} /(2 m S(k))
$$

obeys the Feynman spectrum. Both the Feynman and CBF approximations of $S(k, \omega)$ satisfy these sum rules exactly.

In the analysis of the experiments [18] the frequency dependence of $S(k, \omega)$ was fitted with a Gaussian function. There, the line shift at a given momentum $k$ was defined as the deviation of the maximum of the broad frequency distribution of the measured $S(k, \omega)$ from the free particle value $\hbar^{2} k^{2} /(2 m)$. If we identify that maximum with the average value $\langle\omega(k)\rangle$, we find that the line shift can be calculated directly from $S(k)$ as

$$
\Delta \omega(k)=\frac{\hbar k^{2}}{2 m}\left(\frac{1}{S(k)}-1\right) .
$$

One then concludes that the only information required for a theoretical description of the experimental data is a suitable model interaction and its corresponding static structure factor, which we analyze in the following section.

\section{Feynman Spectrum and the Static Structure Function}

The static structure function, $S(k)$, describes the two-particle distribution function of the ground state in momentum space. In order to get it one needs to solve the ground state wave function of the correlated gas of $N$ Bosonic atoms of mass $m$. The Hamiltonian in the coordinate space,

$$
H=-\frac{\hbar^{2}}{2 m} \sum_{j=1}^{N} \nabla_{j}^{2}+\sum_{1=i<j}^{N} V\left(\left|\mathbf{r}_{i}-\mathbf{r}_{j}\right|\right),
$$

contains the effective, local, two-body potential $V\left(\left|\mathbf{r}_{i}-\mathbf{r}_{j}\right|\right)$, which is the input to the CBF theory. Our aim in this section is to show how sensitive the structure function is to the shape of the two-body potential, assuming that the different potentials tested have the same scattering length.

In the Jastrow method one defines the ground state many-body wave function as a product of two-body correlation factors [23].

$$
\Psi_{0}\left(\mathbf{r}_{1}, \mathbf{r}_{2}, \ldots, \mathbf{r}_{N}\right)=\prod_{1=i<j}^{N} f_{c}\left(\left|\mathbf{r}_{i}-\mathbf{r}_{j}\right|\right),
$$



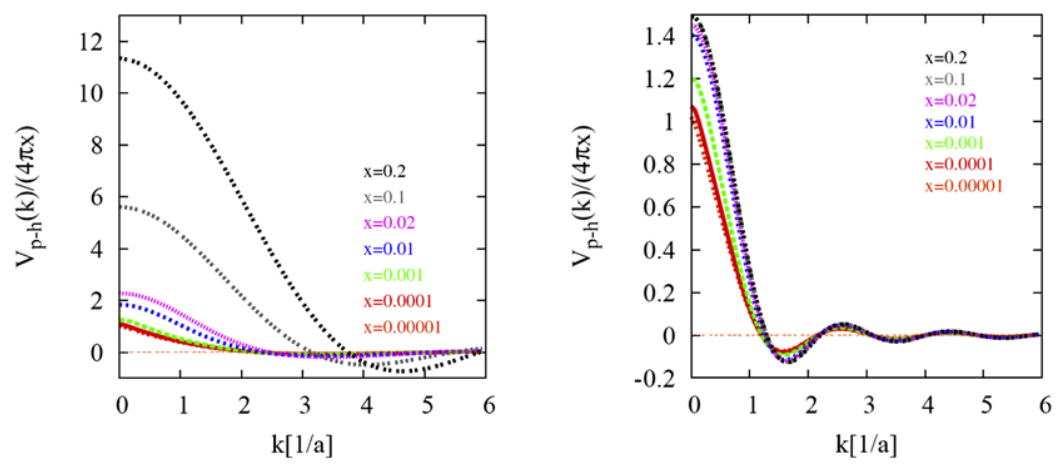

Fig. 4 Effective potentials in momentum space calculated at the values of the gas parameter marked in the figure with color coding. The left and right panels show the results for hard and soft spheres, respectively. Notice the difference in the energy scale (Color figure online)

which defines the pair distribution function $g(r)$ and the structure function in a Bogoliubov-like form [38]

$$
S(k)=\frac{k}{\sqrt{k^{2}+4 \tilde{V}_{\mathrm{p}-\mathrm{h}}(k)}}
$$

written in the scattering length units, where energies and momenta are in units of $\hbar^{2} / m a^{2}$ and $1 / a$, respectively. In this expression, the effective, many-body potential in coordinate space is

$$
V_{p-h}(r)=V(r)+[g(r)-1]\left[w_{\text {ind }}(r)+V(r)\right]+(\nabla \sqrt{g(r)})^{2},
$$

where the bare two-body potential $V(r)$ has been separated from the other terms, which appear due to many-body correlations including the induced potential

$$
\tilde{w}_{\text {ind }}(k)=-\frac{k^{2}}{4}(2 S(k)+1)\left(1-\frac{1}{S(k)}\right)^{2} .
$$

Details of the iterative solutions of these equations are given in Ref. [17].

The effective many-body interaction $\tilde{V}_{\mathrm{p}-\mathrm{h}}(k)$ determines the line shifts completely within the resolution of the experiments as we have discussed before. In Fig. 4 we show its behavior for the hard and soft spheres systems. The range of the hard spheres potential is the scattering length and the soft spheres potential is defined in Eq. (6). The gas parameter covers the range from a very dilute gas with $x=10^{-5}$ to high densities with $x=0.2$ near the solidification of hard spheres. At $x=0.239$ the radius of the one-particle volume

$$
r_{0}=\left(\frac{3}{4 \pi x}\right)^{1 / 3} a
$$

becomes smaller than the radius of the hard sphere and particles touch each other. For the given experimental density $\rho=7.6 \times 10^{13} \mathrm{~cm}^{-3}$ the scattering length values 

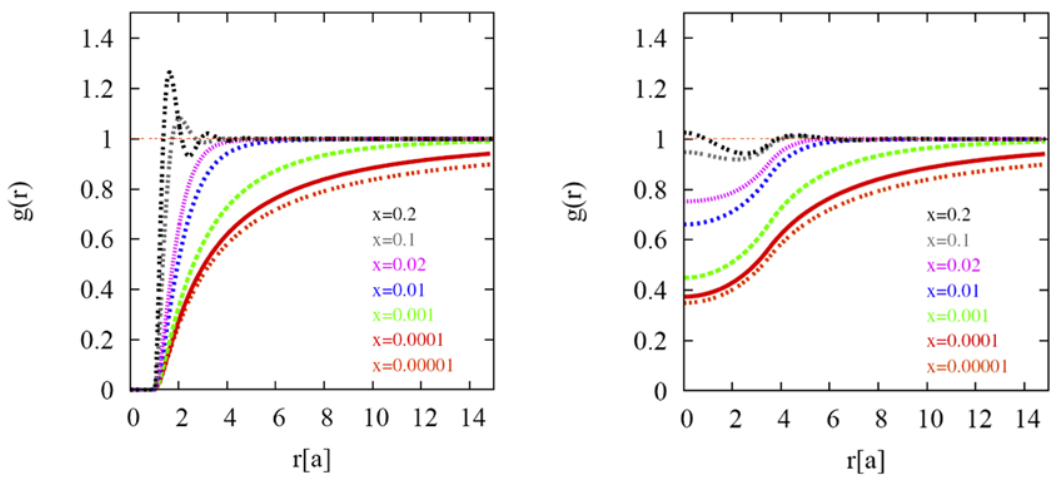

Fig. 5 Two-body radial distribution function for the hard and soft spheres (left and right panels, respectively), calculated at the same values of $x$ as in Fig. 4 (Color figure online)

range from $96.1 a_{0}$ to $2610 a_{0}$, thus spanning a range that is roughly three times the experimental value. In the figure we have divided out the Bogoliubov constant $4 \pi x$ and that is why at $k=0$ the lowest curve is close to unity.

The long wave length limit of the many-body interaction is also known,

$$
\tilde{V}_{\mathrm{p}-\mathrm{h}}(k=0)=m c^{2},
$$

where $c$ is the speed of sound. On the other hand, at small $x$, i.e. $x<10^{-4}$, one recovers the contact potential result $\tilde{V}_{\mathrm{p}-\mathrm{h}}(0)=4 \pi x$ (in units of $\hbar^{2} /\left(m a^{2}\right)$ ). Increasing the scattering length increases the strength of the many-body potential $\tilde{V}_{p-h}(k)$ at $k=0$, because the speed of sound increases. In the case of hard spheres it keeps on increasing with $x$, but in the case of soft spheres it saturates and at the largest values of $x$ $\tilde{V}_{p-h}(k)$ converges to the Fourier transform $\tilde{V}(k)$ of the bare potential. This means that for the soft spheres potential many-body effects in $\tilde{V}_{p-h}(k)$ vanish and therefore the measurements at the largest values available in experiments test directly the Fourier transform of the effective two-body potential. As long as the system remains in the stable gaseous phase. The coherence length where the excitation spectrum becomes single particle like is the point where $4 \tilde{V}_{p-h}(k) \ll k^{2}$. If we take the first zero as that point then the coherence length saturates at $\sim 1.3 a$ in the case of soft spheres, but keeps on increasing with increasing $x$ for hard spheres.

Another measure of the strength of many-body correlations in a quantum system is given by the amount $g(r)$ deviates from unity. In the case of hard spheres $g(r<$ $a)=0$, correlations are always strong and a remarkable, nearest neighbor peak starts to form at large $x$ as shown in the left panel of Fig. 5. The behavior of $g(r)$ in the case of soft spheres is completely different as shown in the right panel of the same figure. It gets closer and closer to 1 with increasing $x$ and many-body correlations vanish, since in this limit $\tilde{V}_{p-h}(k)$ approaches $\tilde{V}(k)$ as mentioned above.

The corresponding structure functions $S(k)$ from Eq. (12) are plotted in Fig. 6. The linear slope at small $k$ is determined by the inverse of the sound velocity, $1 / c$, and therefore decays monotonically to 0 with increasing $x$ for hard spheres while it approaches a constant in the soft spheres case. Furthermore, the structure function 

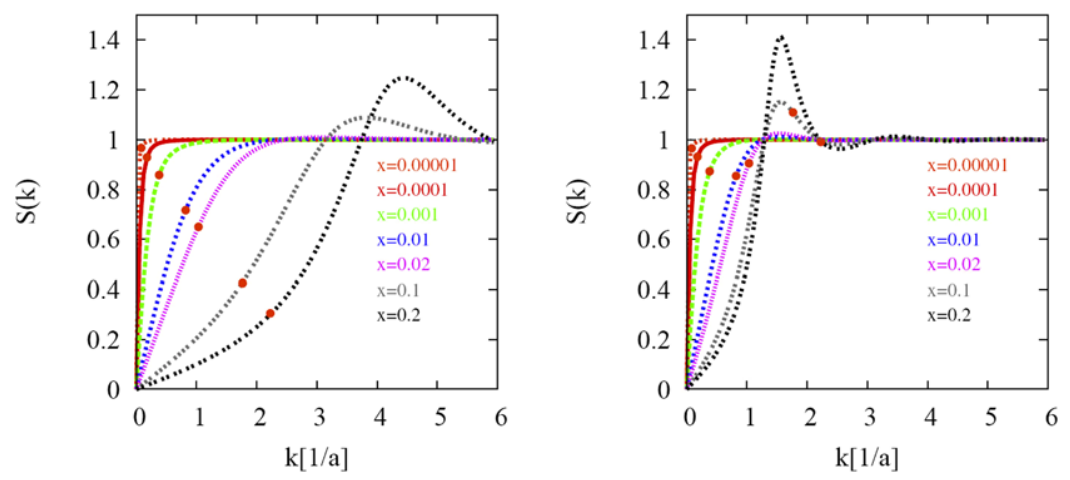

Fig. 6 Static structure functions for the hard and soft spheres (left and right panels, respectively), calculated at the same values of $x$ as in previous figures. The red dots mark the values of the structure functions at experimental momentum $k=4 \pi / 780 \mathrm{~nm}$ (Color figure online)
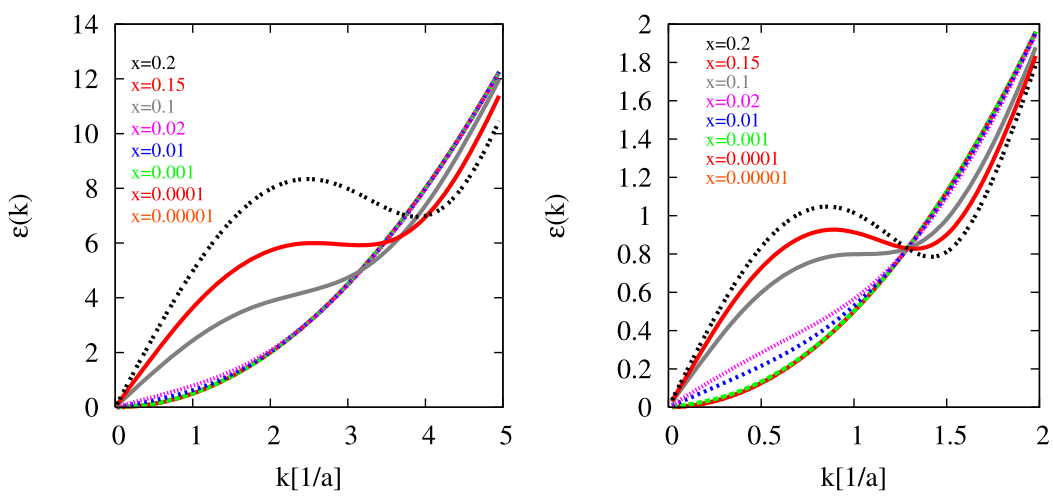

Fig. 7 Excitation spectra for the hard and soft spheres (left and right panels, respectively), calculated at the same values of $x$ as in previous figures. We have also included the curve at $x=0.15$ to show the appearance of the roton minimum (Color figure online)

crosses one at the point $k_{0}$ where $\tilde{V}_{p-h}(k)$ changes sign. For hard spheres $k_{0}$ increases with increasing $x$, while for soft spheres it remains localized in the region $1.1<k_{0}<$ 1.3. In both cases the structure function develops a peak when $x>0.02$ and that is the source of the roton minimum appearing in the excitation spectrum. In $\tilde{V}_{\mathrm{p}-\mathrm{h}}(k)$ that is seen as an attractive minimum.

The red dots shown in Fig. 6 correspond to the experimental momentum value $k=4 \pi / 780 \mathrm{~nm}^{-1}$ in $1 / a$ units. As shown in Eq. (9) the line shift is proportional to $1 / S(k)-1$. In the case of hard spheres this difference increases with increasing $x$ whereas for soft spheres it oscillates. However, actual measurements cover only values up to $x<0.01$ and thus only the first bending is visible in the experiments.

In Fig. 7 we show the Feynman spectra from Eq. (2). As it can be seen from the figure, a roton minimum appears in the soft sphere system at $x \geq 0.1$, while for the hard spheres case the same bending is seen when $x>0.15$. Figure 1 shows the full dynamic structure function $S(k, \omega)$ in the $\mathrm{CBF}$ approximation for hard spheres at 

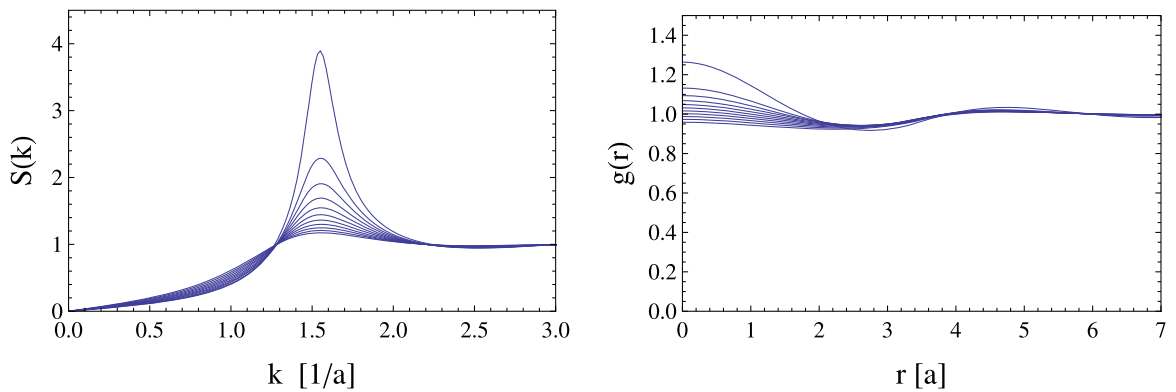

Fig. $8 S(k)$ and $g(r)$ for soft spheres (left and right panels, respectively), calculated for the scattering length values $2130 a_{0}<a<3130 a_{0}$ with $100 a_{0}$ steps. Oscillations increase with increasing $a$ (Color figure online)

Fig. 9 The momentum distribution calculate for the same values of $a$ as in the previous figure. Oscillations increase with increasing $a$ (Color figure online)

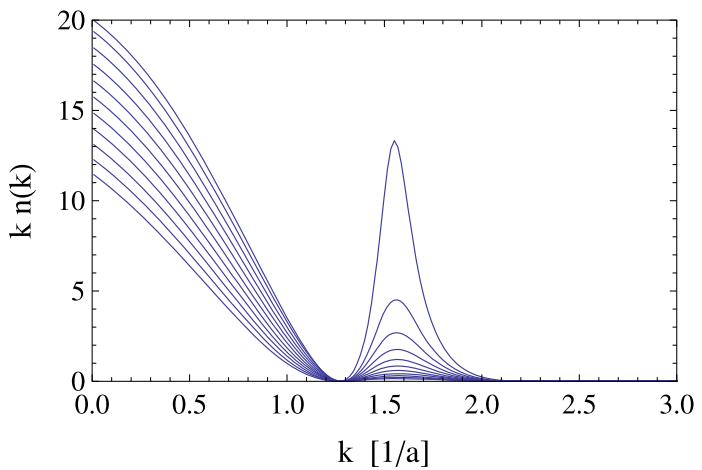

$x=0.1$. The multiphonon contributions push the elementary spectrum down and the roton minimum appears at lower $x$ than in the Feynman spectrum. However and as mentioned before, higher resolution is required to see that in experiments.

In our soft spheres potential many-body correlations diminish up to $x \sim 0.2$. But at larger $x$ there are two interesting suggestions for the phase transition either $g(r)$ at the origin increases rapidly and the first minimum drops to zero indication clusterization [14] or the peak of $S(k)$ becomes singular and the gas becomes unstable against density oscillations. In Fig. 8 we depict $g(r)$ and $S(k)$ for the scattering length values $2130 a_{0}<a<3130 a_{0}$ with $100 a_{0}$ steps corresponding to $0.11<x<0.345$. The upper limits give the critical values of the phase transition where the structure function becomes singular and the wave number of singularity is $k a=1.55$. The singularity appears when the denominator in Eq. (12) vanishes, that is, when

$$
k^{2}+4 \tilde{V}_{p-h}(k)=0 .
$$

As also seen in Fig. 8 the distribution function $g(r)$ remains reasonably flat and its first minimum close to one showing no sign of cluster formation. The density wave instability removes particles from the condensate, because the momentum distribu- 
Fig. 10 The ${ }^{85} \mathrm{Rb}$ line shifts $\Delta \omega / 2 \pi$ from Eq. (9) for different potentials: hard spheres (HS), contact potential (Bogo) and soft spheres with ranges marked in the figure. The red squares with error bars are the Bragg scattering results from Ref. [18] (Color figure online)

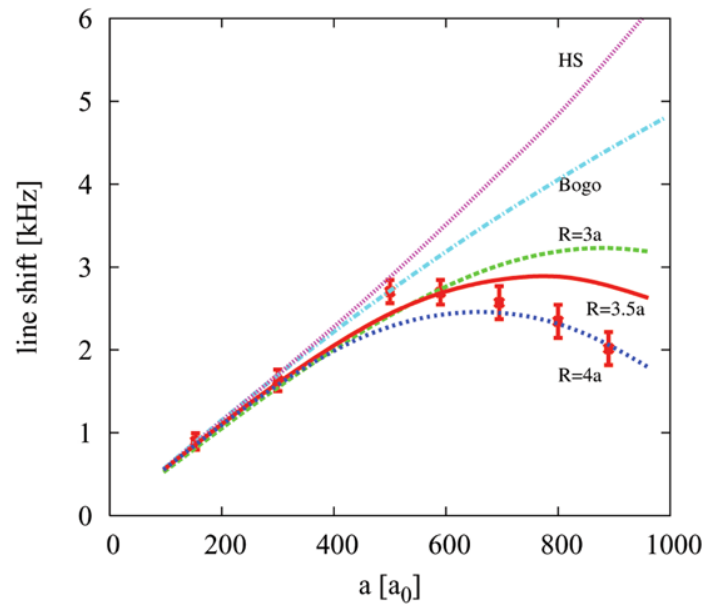

tion [23]

$$
n(k)=4 \frac{(S(k)-1)^{2}}{S(k)}
$$

develops a side peak as shown in Fig. 9. Experimentally that could be easily verified, and that phase transition may be the reason why stable condensates at large scattering length values are very difficult to reach experimentally [18].

In Fig. 10 we compare our results with the actual Bragg scattering experiments. Up to $a=400 a_{0}$ the behavior of the line shift is almost linear and both the mean field result with the contact potential of Eq. (1) and the hard spheres model fit well with the experimental data. Those models, however, are unable to reproduce the strong downwards bending observed in the line shifts at higher values of the scattering length, which comes out reasonably well described with the soft spheres model of Eq. (6). However, a somewhat stronger bending than obtained with the range $R=3.5 a$ is needed. That is achieved by increasing the range as shown in the figure or by adding a weak attraction to the soft spheres repulsion as was done in Ref. [17], but also by using the effective two-body potential derived from the phase shifts of the two-channel Feshbach resonance approach as will be discussed in the next section.

Finally in Fig. 11 we show the behavior of the two-body s-wave scattering phase shift for the soft spheres of Eq. (6) and compare that with the hard spheres and contact potential results. Up to $k a \sim 0.25$ the behavior of all curves is linear and indistinguishable from each other, because $\delta(k)$ is determined by the scattering length alone at low energies. The soft spheres result shows then a minimum at $k a \sim 0.5$ and decaying oscillations at larger $k$. This behavior is determined by fitting the range parameter of the potential to the Bragg scattering results as shown in Fig. 10. It should be compared with the completely different behavior given by the hard spheres system, $\delta(k)=-a k$, and the contact potentials $\delta(k)=-\arctan (k a)$. It is very tempting to identify the upward bending of the phase shift with the downward bending of the line shift as in both cases the hard spheres results lead to bending to different direction. 
Fig. 11 The $s$-wave scattering phase shift of soft spheres (blue solid curve), hard spheres (dashed curve) and the contact potential (dotted curve) (Color figure online)

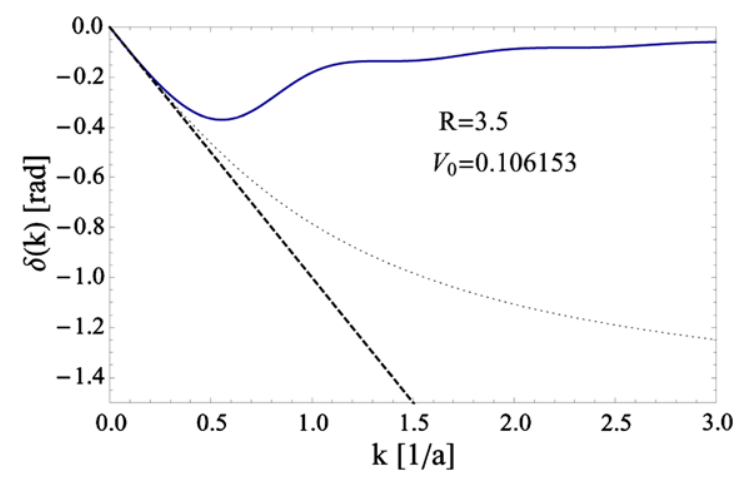

\section{Feshbach Resonance and the Phase Shifts}

In the previous sections we studied properties of two model potentials and showed that the Bragg scattering experiments introduce strong constraints on the shape of the scattering length equivalent potentials. The simple soft spheres model barely survived that test. A slightly better model, the step-well potential, is used in Ref. [17]. These are still to an extend academic models, because the real system contains a weakly bound state, whose energy can be tuned by the magnetic field. In Refs. [17, 22] we solved this Feshbach resonant system and calculated the binding energies and phase shifts for ${ }^{23} \mathrm{Na},{ }^{85} \mathrm{Rb}$ and ${ }^{87} \mathrm{Rb}$ atoms. Here we extend the same model to the ${ }^{133} \mathrm{Cs}$ atoms above the resonance at $B_{0}=-11.7 \mathrm{G}$ where the dimer bound states are derived from experiments. The s-wave phase shifts are then compared with the phase shifts shown in Fig. 11. From the phase shifts we derive the effective potentials at different scattering lengths and use those in the many-body calculations. It will be shown that the soft core potential is a good model in the range of the gas parameter experimentally available at present.

The Feshbach resonance system can be modeled with two coupled Schrödinger equations, one for the bound-state-like closed channel $Q$ and another for the scattering-like open channel state $P$ (for detailed derivations see Refs. [17, 22, 27 , 32, 39]

$$
\begin{aligned}
\left(H_{0}+V^{P}\right)\left|\psi_{k}^{P}\right\rangle+H_{P Q}\left|\psi_{k}^{Q}\right\rangle & =E\left|\psi_{k}^{P}\right\rangle \\
\left(H_{0}+V^{Q}+\epsilon(B)\right)\left|\psi_{k}^{Q}\right\rangle+H_{P Q}\left|\psi_{k}^{P}\right\rangle & =E\left|\psi_{k}^{Q}\right\rangle .
\end{aligned}
$$

The open and closed channel potentials are $V^{P}$ and $V^{Q}$ obtained by projecting out the complete potential to the $P$ and $Q$ subspaces, respectively, while the corresponding states are $\left|\psi_{k}^{P}\right\rangle$ and $\left|\psi_{k}^{Q}\right\rangle . H_{P Q}$ is the coupling Hamiltonian and the energy difference between the uncoupled channels at a given magnetic field is $\epsilon(B)$.

In the Feshbach approximation one ignores the contribution from the continuum and other possible bound states since the leading contribution comes from the bound state near zero in the closed channel [29]. That defines the energy dependent, effective 
open channel potential

$$
U(E)=V^{P}+\frac{H_{P Q}\left|\phi^{Q}\right\rangle\left\langle\phi^{Q}\right| H_{P Q}}{E-E_{\mathrm{res}}}
$$

with $\left|\phi^{Q}\right\rangle$ and $E_{\text {res }}$ the eigenstate and eigenvalue of the uncoupled channel $Q$. With this potential one can solve the Lippmann-Schwinger equation for the open channel T-matrix

$$
T(E)=U(E)+U(E) G_{0}(E) T(E),
$$

where $G_{0}(E)$ is the free particle Green's function. The resonance term in $U(E)$ dominates near $E_{\text {res }}$ and is naturally separable. Since we are interested in the low energy $s$-wave scattering, it is a good approximation to take $\left\langle k\left|V^{P}\right| p\right\rangle=\lambda_{0} f(k) f(p)$ also separable $[17,22,40,41]$. The effective potential in momentum space is now expressed in terms of two momentum dependent functions, $f(k)$ and

$$
\left\langle k\left|H_{P Q}\right| \phi^{Q}\right\rangle=h_{0} h(k)
$$

with the normalization $f(0)=h(0)=1$ and the $\operatorname{limit}_{k \rightarrow \infty} f(k)=\lim _{k \rightarrow \infty} h(k)$ $=0$. As we have very limited information of these function in the intermediate values of momenta we set them equal $h(k)=f(k)[22,32,42]$. The coupling constants $\lambda_{0}$ and $h_{0}$ and the resonance energy $E_{\text {res }}$ are connected to the physical quantities $[17,32]$, the scattering length of the uncoupled open channel $a_{\mathrm{bg}}$, the difference between the magnetic moments of the closed and open channels $\Delta \mu$, the magnetic field $B$ and its resonance value $B_{0}$ and the width of the resonance $\Delta B$. Performing the renormalization the following expression for the on-shell T-matrix is achieved [17],

$$
\langle k|T(E)| k\rangle=\frac{\frac{4 \pi \hbar^{2}}{m} f^{2}(k)}{\frac{1}{a_{b g}\left(1+\frac{\Delta \mu \Delta B}{E-\Delta \mu\left(B-B_{0}\right)}\right)}-\frac{2 m E}{\hbar^{2} \pi} \int_{0}^{\infty} d p \frac{f^{2}(p)}{\frac{m E}{\hbar^{2}}-p^{2}}} .
$$

In the zero energy limit this defines the scattering length through the familiar expression,

$$
a=\frac{m}{4 \pi \hbar^{2}}\langle 0|T(0)| 0\rangle=a_{\mathrm{bg}}\left(1-\frac{\Delta B}{B-B_{0}}\right) .
$$

The pole of the on-shell T-matrix at $E=-E_{b}<0$ defines the two-body binding energy $E_{b}$ as a solution of the equation,

$$
\frac{2}{\pi a} E_{b} \int_{0}^{\infty} \frac{d p f^{2}(p)}{E_{b}+p^{2}}=a_{b g}^{-1}\left(1-\frac{\Delta B}{B-B_{0}+\frac{\hbar^{2} E_{b}}{\Delta \mu m a^{2}}}\right)^{-1},
$$

written in scattering length units. The binding energy $E_{b} \rightarrow 1$ (in units of $\hbar^{2} / m a^{2}$ ) when $a \rightarrow \infty$ and Eq. (25) reduces to an integral condition for $f(p)$,

$$
\frac{2}{\pi} \int_{0}^{\infty} \frac{d p f^{2}(p)}{1+p^{2}}=1
$$


Table 1 Resonance parameters for ${ }^{85} \mathrm{Rb}$ taken from Ref. [43], and parameters for ${ }^{87} \mathrm{Rb},{ }^{23} \mathrm{Na}$ and ${ }^{133} \mathrm{Cs}$ taken from Ref. [44]. Also shown are the fitting parameters $c$ of Eq. (27) from Ref. [22] and this work for ${ }^{133} \mathrm{Cs}$

\begin{tabular}{lccccc}
\hline Atom & $B_{0}(\mathrm{G})$ & $\Delta B(\mathrm{G})$ & $a_{\text {bg }}\left(a_{0}\right)$ & $\Delta \mu\left(\mu_{\mathrm{B}}\right)$ & $c\left(a_{0}\right)$ \\
\hline${ }^{85} \mathrm{Rb}$ & 155.04 & 10.7 & -443 & -2.47 & 60 \\
${ }^{87} \mathrm{Rb}$ & 1007.4 & 0.21 & 100 & 2.79 & 16 \\
${ }^{23} \mathrm{Na}$ & 907 & 1 & 63 & 3.8 & 5 \\
${ }^{133} \mathrm{Cs}$ & -11.7 & 28.7 & 1720 & 2.30 & 62 \\
\hline
\end{tabular}

Fig. 12 Binding energies of the dimer state in ${ }^{133} \mathrm{Cs}$. The red solid curve is the T-matrix result, the black dashed curve gives the $\hbar^{2} / m a^{2}$ behavior and the black dots are the experimental values from Refs. [24, 25] (Color figure online)

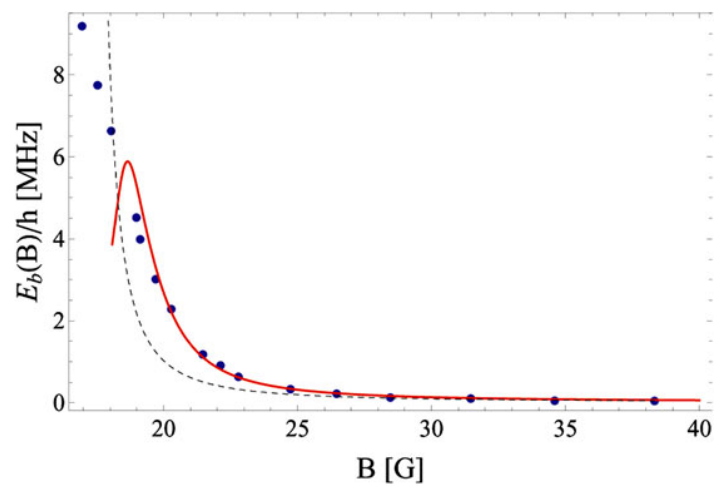

This is the constraint $f(p)$ must satisfy and it cannot be satisfied by a monotonically decreasing $f(p)$.

Following the arguments given in [22] we set

$$
f(p)=\left(1-(1-c / a) p^{2}\right) \exp \left(-p^{2} / K^{2}\right) .
$$

with a Gaussian cut-off and a node at $p_{0}=1 / \sqrt{1-c / a}$. At long wavelengths the effective interaction becomes repulsive and at short wavelengths attractive, supporting a bound state. Equation (26) determines the dimensionless parameter $K=2.586$ and the parameter $c$ is fitted to binding energies $E_{b}$ using Eq. (25).

The experimental resonance parameters $B_{0}, \Delta B, a_{\mathrm{bg}}$ and $\Delta \mu$ taken from Refs. [43, 44] together with the fitted values of the parameter $c$ are shown in Table 1 for different atoms. At the scattering lengths of our main interest $a>200 a_{0}$ the dependence of $f(p)$ on $c$ is weak and vanishes at the unitarity.

The energy of the dimer bound state of ${ }^{133} \mathrm{Cs}$ atoms as a function of magnetic field is experimentally known [24, 25]. The best fit using Eq. (25) is found with $c=62 a_{0}$, which is very close to the ${ }^{85} \mathrm{Rb}$ value $60 a_{0}$. Our results are shown in Fig. 12 and the agreement is very good when $B \geq 18.5 \mathrm{G}$ corresponding to the scattering lengths $a \geq 90 a_{0}$, thus supporting the chosen functional form for $f(p)$.

The on-shell T-matrix of Eq. (23) defines besides the scattering length the full s-wave scattering phase shifts, $\delta(k)$,

$$
\frac{m}{4 \pi \hbar^{2}}\langle k|T(E)| k\rangle=-\frac{1}{k \cot \delta(k)-i k} .
$$




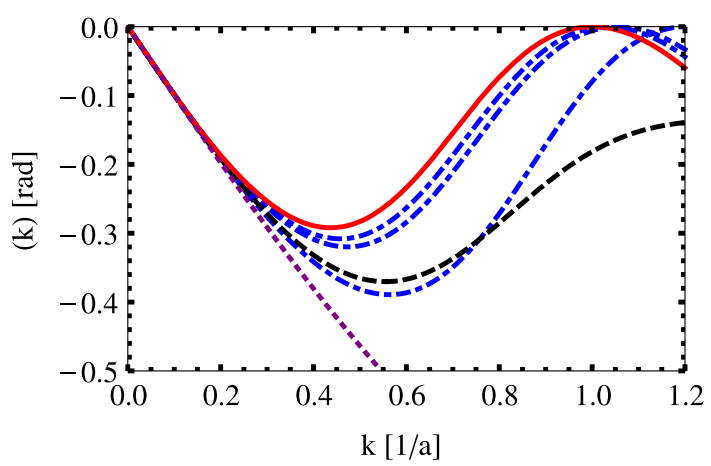

Fig. 13 The s-wave scattering phase shifts for the ${ }^{133} \mathrm{Cs}$ atoms (blue dashed-dotted curves) for scattering lengths $a=200 a_{0}, 600 a_{0}$ and $1000 a_{0}$. The deepest minimum corresponds to the lowest scattering length. The red solid curve gives the phase shift at the unitarity limit. Comparison is made to soft spheres (black dashed curve) and contact potential (dotted purple curve) results (Color figure online)

However, not much is known about the phase shifts beyond the scattering length approximation for the Bose gases analyzed here. The importance of the effective range has been discussed, but at large scattering lengths it is assumed to be small [40]. In Fig. 13 we show our results for ${ }^{133} \mathrm{Cs}$ calculated for scattering lengths $a=$ $200 a_{0}, 600 a_{0}$ and $1000 a_{0}$. In comparison with phase shifts calculated for other atoms in Ref. [22] these results lie on top of the corresponding curves for ${ }^{85} \mathrm{Rb}$ even though the experimental parameters (see Table 1) are very different. At the unitarity limit the s-wave phase shifts for different atoms collapse to the same universal, solid, red line.

These phase shifts are compared with the results from the soft spheres model shown also in Fig. 13. At low momenta $k a<1$ the agreement is reasonable. The difference between the soft spheres and T-matrix results can be traced back to the range parameter $R=3.5 a$ in Eq. (6). By increasing the range of the soft spheres the bending of the phase shift becomes stronger as does the bending in the line shifts in Fig. 10.

If the s-wave phase shift is know for all energies then, if there exists a local, short ranged s-wave potential, it can be uniquely determined by solving the inverse scattering problem. However, any uncertainty in the phase shifts at large energies causes some uncertainty at short distances. We set the phase shift $\delta(p) \equiv 0$ when $p>p_{0}$. That leaves the true short-ranged attraction supporting bound states undetermined, but the long-ranged repulsion contains all the two-body information available to us. The inverse scattering problem is solved by using the Marchenko inversion as discussed in the next section.

\section{Effective Two-Body Potential from the Phase Shifts}

In this section we determine the local, two-body potential $V(r)$ from the known s-wave scattering phase shifts of two identical particles of mass $m$. If the potential is known then the phase shift is determined by the Schrödinger equation for a given 
energy.

$$
-\frac{\hbar^{2}}{m} u^{\prime \prime}(r)+V(r) u(r)=\frac{\hbar^{2} k^{2}}{m} u(r) .
$$

Asymptotically outside the potential range the solution is

$$
u_{l}(r) \sim \sin (k r+\delta(k)) \quad(\text { for large } r),
$$

where $\delta(k)$ is the s-wave phase shift.

Levinson $[45,46]$ proved that if the s-wave phase shifts are known for all energies and no bound states exist then the short-ranged potential is uniquely determined if it exists. The potential must satisfy the following, fairly mild conditions

$$
\begin{aligned}
& \int_{0}^{\infty} r|V(r)| d r<\infty, \\
& \int_{0}^{\infty} r^{2}|V(r)| d r<\infty .
\end{aligned}
$$

The method we have chosen to use in the calculation of the potential is based on the Marchenko integral equation and expansion of the phase shift in rational series $[33,34]$. To summarize that method briefly, we first recall that the S-matrix is fully characterized by the phase shift, $S_{0}(k)=\exp [2 i \delta(k)]$. By using the S-matrix and the outgoing s-wave Riccati-Hankel functions $h_{0}^{+}(z)$, one defines the input kernel

$$
F(r, s)=\frac{1}{2 \pi} \int_{-\infty}^{\infty} h_{0}^{+}(k r)\left[1-S_{0}(k)\right] h_{0}^{+}(k s) d k,
$$

which is then inserted into the Marchenko integral equation

$$
K(r, s)+F(r, s)+\int_{r}^{\infty} K(r, t) F(t, s) d t=0 .
$$

From the solution, $K(r, s)$, of this equation one calculates the potential through the relation

$$
V(r)=-2 \frac{d}{d r} K(r, r)
$$

That particular method has two parameters as explained in the Appendix. The first parameter $N$ is the order of the rational representation of the phase shift and the second one is the order of the Padé approximate denoted by $L$. We chose to use the values $N=12$ and $L=4$, which give the desired accuracy of the rational polynomial fitting to the input phase shift with the difference being less than $5 \cdot 10^{-4} \mathrm{rad}$. The procedure is also checked by inserting the obtained potential into the Schrödinger equation and solving that for the phase shifts. The results are exactly the same within the numerical accuracy. A typical fit is shown in Fig. 14.

The input to the Marchenko inversion is the s-wave phase shift calculated from the on-shell T-matrix $\langle k|T(E)| k\rangle$ of Eq. (23) using Eq. (28). From Eq. (23) it is easy to 
Fig. 14 A typical input phase shift into the Marchenko inversion (red dots) taken from Fig. 13 at unitarity. Solid line is the rational function fit to the $\delta(k)$ of the form (37) in the Appendix with $N=12$ where the coefficients $a_{2 n-1}$ and $b_{2 n}$ were determined by using the method of least squares (Color figure online)

Fig. 15 The universal, effective potential from the T-matrix approach (full red curve) compared to the soft spheres potentials (dashed curves) with the range parameters marked in the figure (Color figure online)
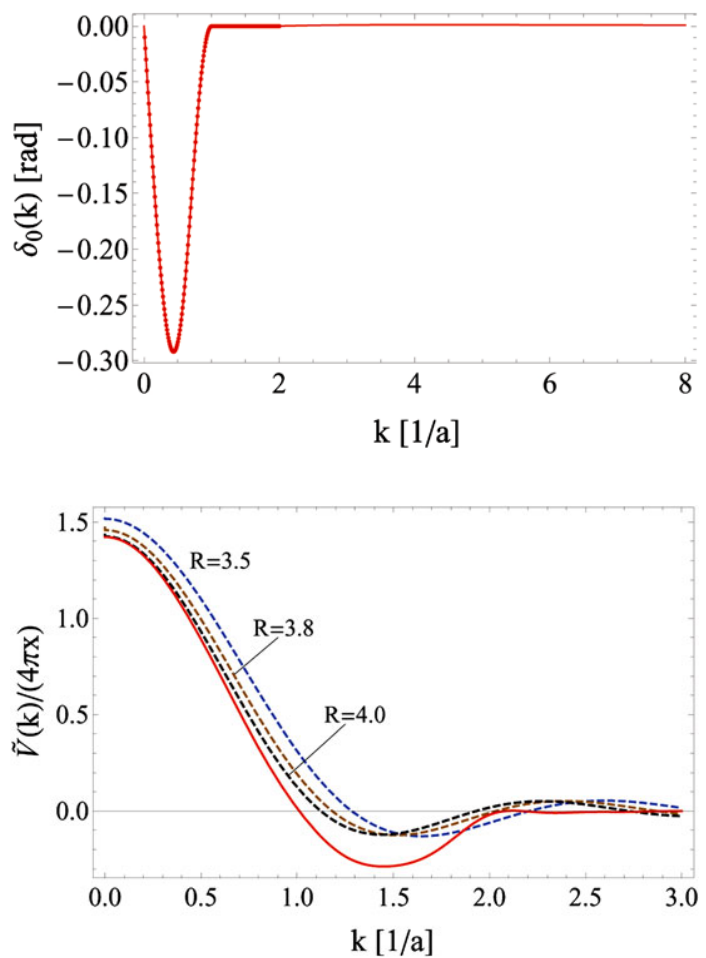

see that $\langle k|T(E)| k\rangle$ vanishes if the cutoff-function $f(k)$ vanishes, which happens at $k=p_{0}$. According to Eq. (28), this means that

$$
\delta\left(p_{0}\right)=0 \quad(\bmod \pi) .
$$

We construct as an example the effective potential, which gives the same s-wave phase shift up to $k=p_{0}$ as in the unitarity limit and remains very small for $k>p_{0}$ as shown in Fig. 14. This separates the long wavelength repulsion from the short wavelength attraction and removes the bound state [22]. The low energy, many-body properties depend, however, very little on the details in the short-ranged attraction of the effective potential. It is therefore more illuminating to look at the effective potential in momentum space shown in Fig. 15 with the red solid curve. It displays clearly the long wavelength repulsion followed by an intermediate range attraction. This behavior agrees very well with the Fourier transform of the soft spheres potentials calculated for three different range parameters $R=3.5 a, 3.8 a$ and $4.0 a$ as show in Fig. 15.

With the T-matrix effective potentials obtained by the above procedure we have calculated the Bragg scattering line shifts shown in Fig. 16 for ${ }^{85} \mathrm{Rb},{ }^{23} \mathrm{Na},{ }^{87} \mathrm{Rb}$ and ${ }^{133} \mathrm{Cs}$. In the ${ }^{85} \mathrm{Rb}$ case the agreement with experiments is very good and the ${ }^{133} \mathrm{Cs}$ curve is almost indistinguishable from that. The results for the ${ }^{23} \mathrm{Na},{ }^{87} \mathrm{Rb}$ lie somewhat lower, but all these line shifts are very similar, because the underlying effective two-body potentials and the resulting structure functions are very close to each other. 


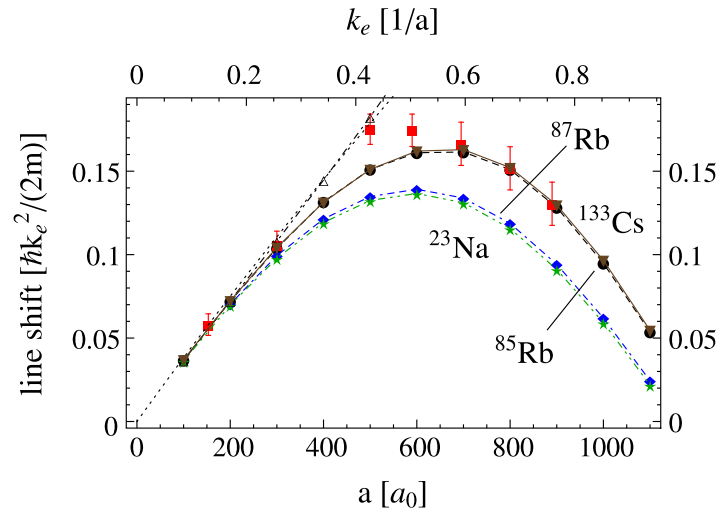

Fig. 16 Line shifts $\hbar \Delta \omega(k)$ for ${ }^{85} \mathrm{Rb}$ (black circles), ${ }^{87} \mathrm{Rb}$ (blue diamonds), ${ }^{133} \mathrm{Cs}$ (brown triangles) and ${ }^{23} \mathrm{Na}$ (green stars) as a function of the scattering length. The squares with error bars are the Bragg scattering results from Ref. [18]. Also shown are the hard sphere (dashed-dotted curve) and the contact potential (dotted curve) results. The top axis shows the experimental momentum $k_{e}=4 \pi / 780 \mathrm{~nm}^{-1}$ in $1 / a$ units and the frequency unit is $\hbar k_{e}^{2} / 2 m$ (Color figure online)

The parametrization of soft spheres potential contains one free parameter fixed by the Bragg scattering. The longest range $R=4 a$ fits better the ${ }^{23} \mathrm{Na}$ and ${ }^{87} \mathrm{Rb}$ line shifts and $R=3.5 a$ is reasonable for ${ }^{85} \mathrm{Rb}$ and ${ }^{133} \mathrm{Cs}$ atoms. Yet, the bending is not strong enough as shown in Fig. 10. The step-well model studied in Ref. [17] with more fitting parameters gives a better agreement. In the case of the T-matrix momentum space potentials there are no free parameters after the fitting of the dimer energies. This shows how intimately the many-body excitations and two-body scattering results are related to each other in cold Bose gases.

As we showed in Sect. 3 a two-body potential that is attractive at intermediate momenta and together with the many-body corrections satisfies Eq. (17) will cause the density wave instability. The evolution of that instability using the T-matrix potential is depicted in Fig. 17 where we show both $S(k)$ and $g(r)$ for the values of scattering length starting from $1550 a_{0}$ to $2050 a_{0}$ with $50 a_{0}$ steps. In terms of the gas parameters the range is $0.04<x<0.095$. The curve with $a=2050 a_{0}$ is clearly in the unstable region and does not correspond to a consistent solution. In Fig. 18 we show the evolution of the maxon-roton shape into the instability and the growth of the side peak in momentum distribution using the same values of $a$. We then conclude that using a realistic T-matrix potential the instability happens at $x=0.09$ corresponding to the scattering length $a \sim 2000 a_{0}$ and $k a=1.3$. This is already temptingly close to the conditions which are experimentally reachable.

\section{Conclusions}

In summary, we have shown that the Bragg scattering experiments bring in a sensitive test to the shape of the scattering length equivalent two-body potentials, ruling out the hard spheres and contact potential models and favoring the soft spheres ones. In order 

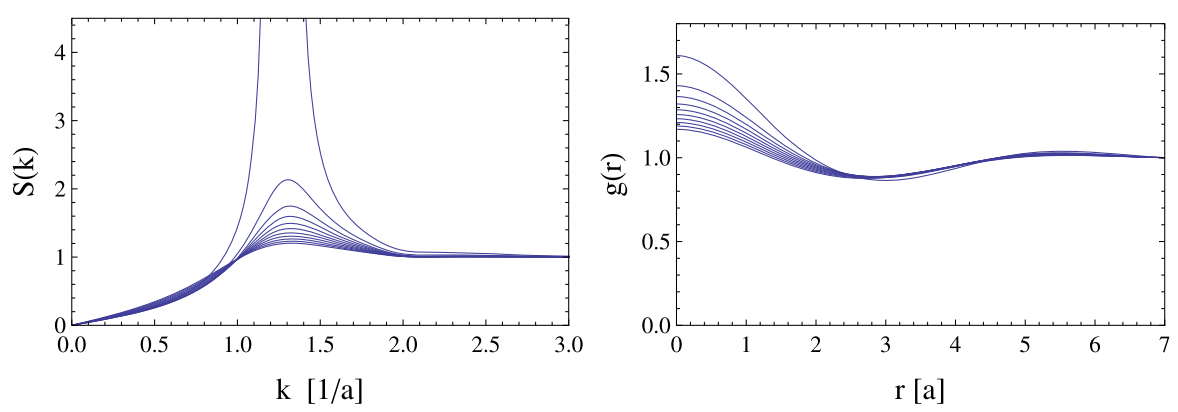

Fig. $17 S(k)$ and $g(r)$ for the T-matrix potential (left and right panels, respectively), calculated for the scattering length values $1550 a_{0}<a<2050 a_{0}$ with $50 a_{0}$ steps. Oscillations increase with increasing $a$ (Color figure online)
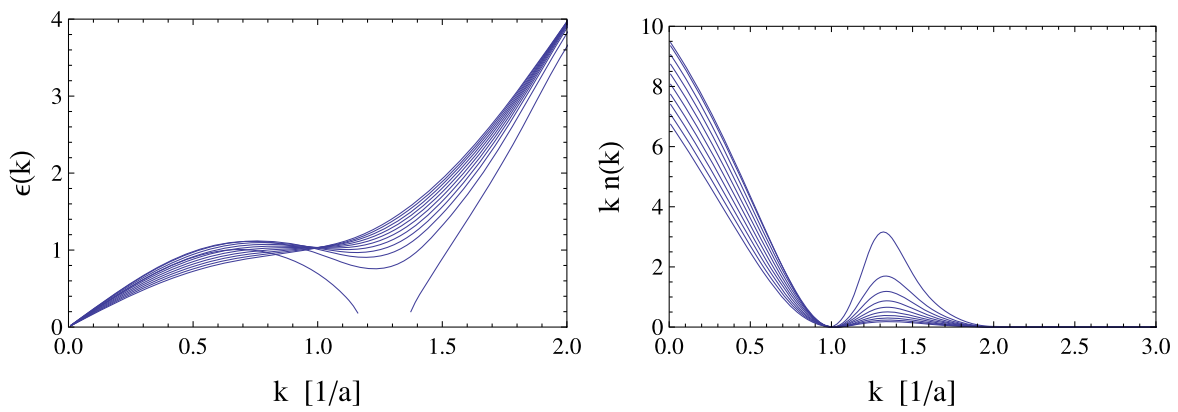

Fig. 18 The excitation spectra and momentum distributions (left and right panels, respectively) calculate for the same values of $a$ as in Fig. 17. Oscillations increase with increasing $a$ (Color figure online)

to make connection from these model potentials to the realistic systems with a Feshbach resonance we have also solved the set of two coupled Schrödinger equations in the Feshbach resonance approximation using the separable T-matrix assumption and derived the effective two-particle potential. That potential is fixed by experimental quantities like magnetic field, resonance width, two-body bound state etc. In comparison to the model potentials the T-matrix potential is very close to the soft spheres model in momentum space. Thus it gives theoretical support for the use of such an academic model in many-body calculations. By construction the T-matrix potential becomes universal in the unitarity limit.

We have also shown that both the T-matrix and soft spheres potential give rise to the density wave instability. The condition for the instability states that the manybody potential $\tilde{V}_{p-h}(k) \leq-k^{2} / 4$ in scattering length units. The phase transition happens even though two- and higher-body bound states are removed from the effective interaction. The critical value of the gas parameter is $x \sim 0.09$. Experimentally it shows up as a side peak in the momentum distribution. Our results show that softening of the roton minimum is a generic phenomenon in all Feshbach resonant Bose condensates. What we have seen is the second order phase transition, but is there a first order phase transition preceding that, which organizes the superfluid into the supersolid phase, remains to be studied. 
Acknowledgements We thank V. Apaja, G. Astrakharchik, J. Boronat and A. Polls for discussions. This work has been supported by Grant No. FIS2008-0443 from DGI (Spain), and Grant No. 2009-SGR1003 from the Generalitat de Catalunya. One of us (R.S.) thanks the Finnish Cultural Foundation and Vilho, Yrjö and Kalle Väisälä fund for financial support.

\section{Appendix: Marchenko inversion}

Our method to solve Marchenko equations (33) and (34) follows the presentation in Ref. [34]. That method is based on a rational function representation of the phase shift data. That is, first one fits the phase shift data with a rational function

$$
\delta(k)=\frac{\sum_{n=1}^{N / 2} a_{2 n-1} k^{2 n-1}}{1+\sum_{n=1}^{N / 2} b_{2 n} k^{2 n}},
$$

where $N \in\{2,4,6, \ldots\}$. The next step is to cast the S-matrix $S_{0}(k)=\exp \left[2 i \delta_{l}(k)\right]$ into a rational form. That can be done by using the Padé approximation

$$
e^{z}=\frac{\sum_{l=0}^{L} a_{l} z^{l}}{\sum_{l=0}^{L} a_{l}(-z)^{l}}=1+\sum_{l=1}^{L} \frac{E_{l}}{z-\epsilon_{l}} .
$$

The coefficient $a_{l}=(2 L-l) ! /[l !(L-l) !]$. The constants $\left\{\epsilon_{1}, \ldots, \epsilon_{L}\right\}$ are roots of the polynomial appearing in the denominator, and they appear in conjugate complex pairs. Applying the Padé approximation (38) to $S_{0}(k)$, one obtains

$$
S_{0}(k)=\frac{\sum_{l=0}^{L} a_{l}[2 i \delta(k)]^{l}}{\sum_{l=0}^{L} a_{l}[-2 i \delta(k)]^{l}}=1-\sum_{m=1}^{M} \frac{S_{m}}{k-\sigma_{m}},
$$

where $\sigma_{m}$ are roots of the denominator. That is, roots of the equations $2 i \delta(k)=\epsilon_{l}$, where $l \in\{1, \ldots, L\}$. Since $\delta(k)$ is now a rational function, these equations are easy to solve. Moreover, since the order of $\delta(k)$ is $N$, the total number of roots $\sigma_{m}$ is $M=L \times N$.

Let us now assume that $L$ is even. Then the $S$-matrix can be written as a rational polynomial,

$$
S_{0}(k)=\frac{\prod_{i=1}^{M}\left(k+\sigma_{i}\right)}{\prod_{i=1}^{M}\left(k-\sigma_{i}\right)} .
$$

By matching Eq. (39) to Eq. (40) at $k=\sigma_{m}$, one obtains the pole strengths

$$
S_{m}=-2 \sigma_{m} \prod_{\substack{i=1 \\ i \neq m}}^{M} \frac{\sigma_{m}+\sigma_{i}}{\sigma_{m}-\sigma_{i}}
$$

By using Eq. (39), one casts the input kernel (33) into the form

$$
F(r, s)=\frac{1}{2 \pi} \int_{-\infty}^{\infty} h_{l}^{+}(k r)\left[\sum_{m=1}^{M} \frac{S_{m}}{k-\sigma_{m}}\right] h_{l}^{+}(k s) d k
$$




$$
=i \sum_{m}^{(+)} S_{m} h_{l}^{+}\left(\sigma_{m} r\right) h_{l}^{+}\left(\sigma_{m} s\right)
$$

where $\sum_{m}^{(+)}$means summation over the poles located in the upper half of the complex plane. For the output kernel one uses the ansatz

$$
K(r, s)=\sum_{m}^{(+)} \Psi(m, r) h_{l}^{+}\left(\sigma_{m} s\right) .
$$

By substituting Eqs. (42) and (43) into the Marchenko equation (34), one obtains

$$
\sum_{m}^{(+)} h_{l}^{+}\left(\sigma_{m} s\right)\left[\Psi(m, r)+i S_{m} h_{l}^{+}\left(\sigma_{m} r\right)+i S_{m} \sum_{n}^{(+)} M_{m, n}(r) \Psi(n, r)\right]=0,
$$

where

$$
M_{m, n}(r)=\int_{r}^{\infty} h_{l}^{+}\left(\sigma_{m} t\right) h_{l}^{+}\left(\sigma_{n} t\right) d t
$$

The linear independence of the Riccati-Hankel functions, $h_{l}^{+}\left(\sigma_{m} s\right)$, implies a system of $M / 2$ linear equations for the $\Psi(n, r)$,

$$
\sum_{n}^{(+)}\left[\delta_{m n}+i S_{m} M_{m, n}(r)\right] \Psi(n, r)=-i S_{m} h_{l}^{+}\left(\sigma_{m} r\right) .
$$

It is easy to solve numerically the values $\Psi(n, r)$ for any fixed $r$. After that, one obtains the output kernel $K(r, s)$ by substituting the functions $\Psi(n, r)$ into Eq. (43). Subsequently, the potential $V(r)$ can be calculated by using Eq. (35).

\section{References}

1. T.D. Lee, C.N. Yang, Phys. Rev. 105, 1119 (1957)

2. T.D. Lee, K. Huang, C.N. Yang, Phys. Rev. 106, 1135 (1957)

3. D.M. Stamper-Kurn, A.P. Chikkatur, A. Görlitz, S. Inouye, S. Gupta, D.E. Pritchard, W. Ketterle, Phys. Rev. Lett. 83, 2876 (1999)

4. J. Steinhauer, R. Ozeri, N. Katz, N. Davidson, Phys. Rev. Lett. 88, 120407 (2002)

5. R. Ozeri, N. Katz, J. Steinhauer, N. Davidson, Rev. Mod. Phys. 77, 187 (2005)

6. P.T. Ernst, S. Götze, J.S. Krause, K. Pyka, D.S. Lühmann, D. Pfankuche, K. Sengstock, Nat. Phys. (2008). Published online. doi:10.1038/nphys1505

7. E.A. Kolganova, A.K. Motovilov, W. Sandhas, Phys. Rev. A 70, 052711 (2004)

8. P. Grüter, D. Ceperley, F. Laloë, Phys. Rev. Lett. 79, 3549 (1997)

9. J.D. Reppy, B.C. Crooker, B. Hebral, A.D. Corwin, J. He, G.M. Zassenhaus, Phys. Rev. Lett. 84, 2060 (2000)

10. S.C. Cormack, D. Schumayer, D.A.W. Hutchinson, Phys. Rev. Lett. 107, 140401 (2011)

11. L.P. Pitaevskii, JETP Lett. 39, 511 (1984)

12. D.H.J. O’Dell, S. Giovanazzi, G. Kurizki, Phys. Rev. Lett. 90, 110402 (2003)

13. D. Hufnagl, R. Kaltseis, V. Apaja, R.E. Zillich, Phys. Rev. Lett. 107, 065303 (2011)

14. F. Cinti, P. Jain, M. Boninsegni, A. Micheli, P. Zoller, G. Pupillo, Phys. Rev. Lett. 105, 135301 (2010) 
15. S. Saccani, S. Moroni, M. Boninsegni, Phys. Rev. Lett. 108, 175301 (2012)

16. M. Boninsegni, J. Low Temp. Phys. 1-13 (2012)

17. R. Sarjonen, M. Saarela, F. Mazzanti, Eur. Phys. J. D 65, 25 (2011)

18. S.B. Papp, J.M. Pino, R.J. Wild, S. Ronen, C.E. Wieman, D.S. Jin, E.A. Cornell, Phys. Rev. Lett. 101, $135301(2008)$

19. S.C. Cormack, D. Schumayer, D.A.W. Hutchinson, Phys. Rev. Lett. 107, 140401 (2011)

20. S.T. Beliaev, J. Exp. Theor. Phys. 7, 289 (1958)

21. S. Ronen, J. Phys. B, At. Mol. Opt. Phys. 42, 055301 (2009)

22. R. Sarjonen, M. Saarela, F. Mazzanti, Phys. Rev. A 84, 041602 (2011)

23. E. Feenberg, Theory of Quantum Fluids (Academic, New York, 1969)

24. M. Mark, F. Ferlaino, S. Knoop, J.G. Danzl, T. Kraemer, C. Chin, H.C. Nägerl, R. Grimm, Phys. Rev. A 76, 042514 (2007)

25. M. Jona-Lasinio, L. Pricoupenko, Phys. Rev. Lett. 104, 023201 (2010)

26. A.J. Leggett, Rev. Mod. Phys. 73, 307 (2001)

27. C.J. Pethick, H. Smith, Bose-Einstein Condensation in Dilute Gases (Cambridge University Press, Cambridge, 2002)

28. T. Köhler, K. Góral, P.S. Julienne, Rev. Mod. Phys. 78, 1311 (2006)

29. H. Feshbach, Ann. Phys. 19, 287 (1962)

30. C.C. Chang, C.E. Campbell, Phys. Rev. B 13, 3779 (1976)

31. E. Krotscheck, M. Saarela, Phys. Rep. 232, 1 (1993)

32. S.J.J.M.F. Kokkelmans, J.N. Milstein, M.L. Chiofalo, R. Walser, M.J. Holland, Phys. Rev. A 65, 053617 (2002)

33. Z.S. Agranovich, V.A. Marchenko, The Inverse Problem of Scattering Theory (Gordon, New York, 1964)

34. T. Kirst, K. Amos, L. Berge, M. Coz, H.V. von Geramb, Phys. Rev. C 40, 912 (1989)

35. V. Apaja, J. Halinen, V. Halonen, E. Krotscheck, M. Saarela, Phys. Rev. B 55, 12925 (1997)

36. F. Mazzanti, A. Polls, A. Fabrocini, Phys. Rev. A 67, 063615 (2003)

37. S. Giorgini, J. Boronat, J. Casulleras, Phys. Rev. A 60, 5129 (1999)

38. E. Krotscheck, Phys. Rev. B 33, 3158 (1986)

39. A.J. Leggett, Quantum Liquids: Bose Condensation and Cooper Pairing in Condensed-Matter Systems (Oxford University Press, London, 2007)

40. G.M. Bruun, A.D. Jackson, E.E. Kolomeitsev, Phys. Rev. A 71, 052713 (2005)

41. G.E. Brown, A.D. Jackson, The Nucleon-Nucleon Interaction (North-Holland, Amsterdam, 1976)

42. L. Pricoupenko, M. Jona-Lasinio, Phys. Rev. A 84, 062712 (2011)

43. T. Köhler, T. Gasenzer, P. Julienne, K. Burnett, Phys. Rev. Lett. 91, 230401 (2003)

44. C. Chin, R. Grimm, P. Julienne, E. Tiesinga, Rev. Mod. Phys. 82, 1225 (2010)

45. N. Levinson, Kgl. Danske Videnskab Selskab Mat.-fys. Medd. 25 (1949)

46. R. Jost, W. Kohn, Phys. Rev. 87, 977 (1952) 